\title{
Exome sequencing reveals CHM mutations in six families with atypical choroideremia initially diagnosed as retinitis pigmentosa
}

\author{
SHIQIANG LI ${ }^{1 *}$, LIPING GUAN ${ }^{2 *}$, SHAOHUA FANG $^{1}$, HUI JIANG $^{2}$, XUESHAN XIAO $^{1}$, JIANHUA YANG $^{2}$, \\ PANFENG WANG ${ }^{1}$, YE YIN ${ }^{2}$, XIANGMING GUO ${ }^{1}$, JUN WANG $^{2}$, JIANGUO ZHANG $^{2 *}$ and QINGJIONG ZHANG ${ }^{1^{*}}$ \\ ${ }^{1}$ State Key Laboratory of Ophthalmology, Zhongshan Ophthalmic Center, Sun Yat-sen University, \\ Guangzhou, Guangdong 510060; ${ }^{2}$ BGI-Shenzhen, Shenzhen, Guangdong 518083, P.R. China
}

Received February 14, 2014; Accepted May 30, 2014

DOI: $10.3892 /$ ijmm.2014.1797

\begin{abstract}
Mutations in almost 200 genes are associated with hereditary retinal diseases. Of these diseases, retinitis pigmentosa (RP) is the most common and is genetically and clinically highly heterogeneous. At least 62 genes are associated with $\mathrm{RP}$ and mutations in these genes account for approximately half of the cases of disease. In the present study, mutations in the $C H M$ gene, which are known to associate with choroideremia, were identified in six of 157 families with retinitis pigmentosa by whole exome sequencing. No potential pathogenic mutations in the $62 \mathrm{RP}$-associated genes were found in the six families. Sanger sequencing confirmed the mutations in $C H M$, including four novel (c.558_559delTT, c.964G>T, c.966delA, c.1166+2T>G) and two known (c.703-1G>A and c.1584_1587delTGTT) mutations. Available clinical data suggest an atypical phenotype of choroideremia in these patients compared to that of Caucasians. Overlapping clinical features and atypical phenotypic variation may contribute to the confusion of one another. Awareness of the phenotypic variation and careful clinical examination may facilitate proper clinical diagnosis and genetic counseling of complicated hereditary retinal diseases. Whole exome sequencing therefore is useful in the identification of genetic cause for less clarified hereditary retinal diseases and enriches our understanding of phenotypic variations of gene mutation.
\end{abstract}

\section{Introduction}

Hereditary retinal degeneration is the most common form of genetic eye diseases causing irreversible blindness.

Correspondence to: Dr Qingjiong Zhang, State Key Laboratory of Ophthalmology, Zhongshan Ophthalmic Center, Sun Yat-sen University, 54 Xianlie Road, Guangzhou, Guangdong 510060, P.R. China

E-mail: zhangqji@mail.sysu.edu.cn; qingjiongzhang@yahoo.com

*Contributed equally

Key words: whole exome sequencing, mutations, CHM, retinitis pigmentosa, choroideremia
Mutations in almost 200 genes are associated with hereditary retinal degeneration (RetNet: http://www.sph.uth.tmc. edu/Retnet/sum-dis.htm). Of these diseases, retinitis pigmentosa (RP) is the most common type and refers to various forms of progressive retinal degeneration with predominantly impaired rod photoreceptors. RP is clinically and genetically highly heterogeneous. Currently, mutations in at least 62 genes were associated with autosomal dominant, autosomal recessive, or X-linked RP. Mutations in all these genes may be responsible for approximately half of the cases of RP (1-3). The causes for the remaining half of RP cases await identification. The development of high-throughput techniques for gene analysis has revealed an increasing number of new genes that are associated with RP $(4,5)$. Additionally, a number of genes known to cause other retinopathy or extraocular diseases have been recently reported to also be responsible for RP, such as mutation in ADAMTS18, CYP4V2, or OFD1 (6-8).

In the present study, exome sequencing on 157 families with RP detected mutations in the RAB geranylgeranyl transferase holoenzyme component A gene (the CHM gene) in six families. These mutations are known to cause choroideremia (9-11). No other causative mutations in the 61 of the 62 RP-associated genes were detected in the six families by exome sequencing. Sanger sequencing confirmed four novel and two known mutations in the $C H M$ gene in the six families. Clinical data of the six probands showed certain characteristics of RP and some atypical indications of choroideremia. The result emphasizes the importance of a comprehensive analysis of clinical data and potential genes for hereditary diseases with overlapping phenotypes (12) or among the list requiring differential diagnosis.

\section{Materials and methods}

Patients. Probands with initial diagnosis of RP from 157 families were recruited at the Eye Hospital of the Zhongshan Ophthalmic Center (Guangzhou, China). The diagnosis of RP was based on night blindness beginning in early childhood, decreasing visual acuity with age, and fundus changes as previously described (13). Written informed consent that followed the tenets of the Declaration of Helsinki was obtained from each participating individual or their guardians prior to the study. This study was approved by the Institutional Review 
Table I. Primers used for mutation confirmation by Sanger sequencing for the $C H M$ gene.

\begin{tabular}{|c|c|c|c|}
\hline Proband & Primers and sequences $\left(5^{\prime} \rightarrow 3^{\prime}\right)$ & Size of amplicons (bp) & Annealing temp $\left({ }^{\circ} \mathrm{C}\right)$ \\
\hline RP31 & $\begin{array}{l}\text { F1: atggatcaggttttgctgct } \\
\text { R1: aagctgatgeccagttacaa }\end{array}$ & 397 & $58-65$ \\
\hline RP229 & $\begin{array}{l}\text { F2: ctgcctacggaggatgagtc } \\
\text { R2: gggcccagatactgttttca }\end{array}$ & 337 & $58-65$ \\
\hline RP263 & $\begin{array}{l}\text { F3: aattaaccccaacctccaa } \\
\text { R3: aagctcaaaaagaggccaca }\end{array}$ & 383 & $58-65$ \\
\hline RP285, RP304 & $\begin{array}{l}\text { F4: ccacctatgtcetttgtgagg } \\
\text { R4: aatggagtgttgccataccg }\end{array}$ & 291 & $58-65$ \\
\hline RP359 & $\begin{array}{l}\text { F5: caccatgacttgctcagctc } \\
\text { R5: cccacatgtttaggcagaca }\end{array}$ & 376 & $58-65$ \\
\hline
\end{tabular}

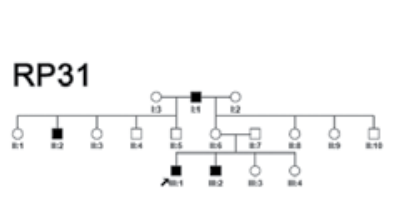

RP229
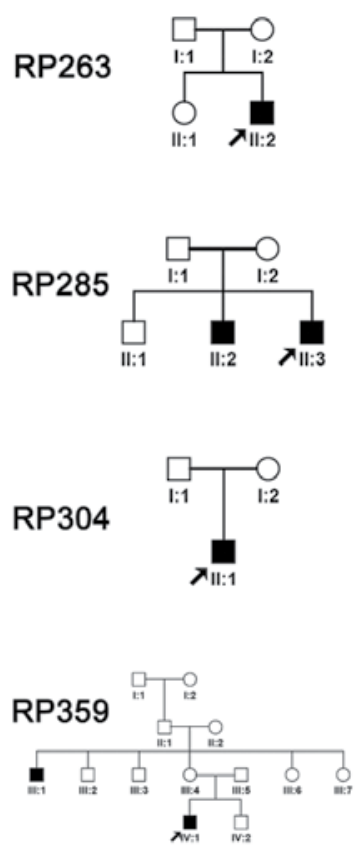

\section{Mutant sequence}

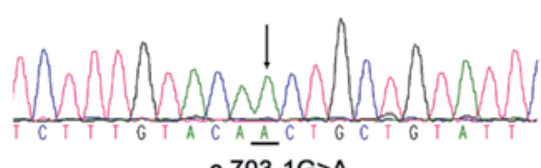

c. $703-1 \mathrm{G}>\mathrm{A}$

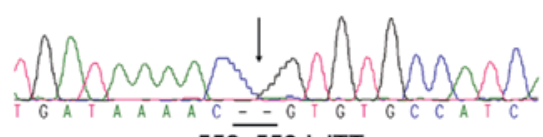

c.558_559delTT
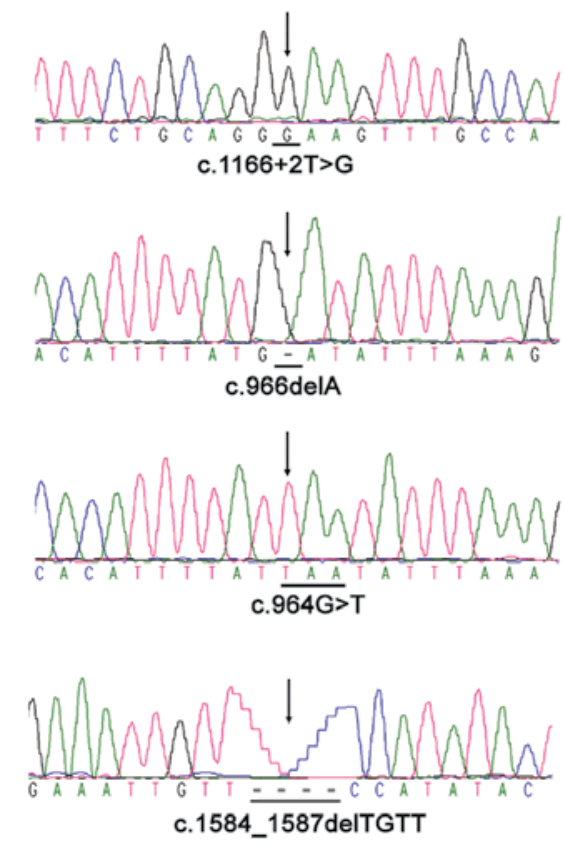

\section{Normal sequence}
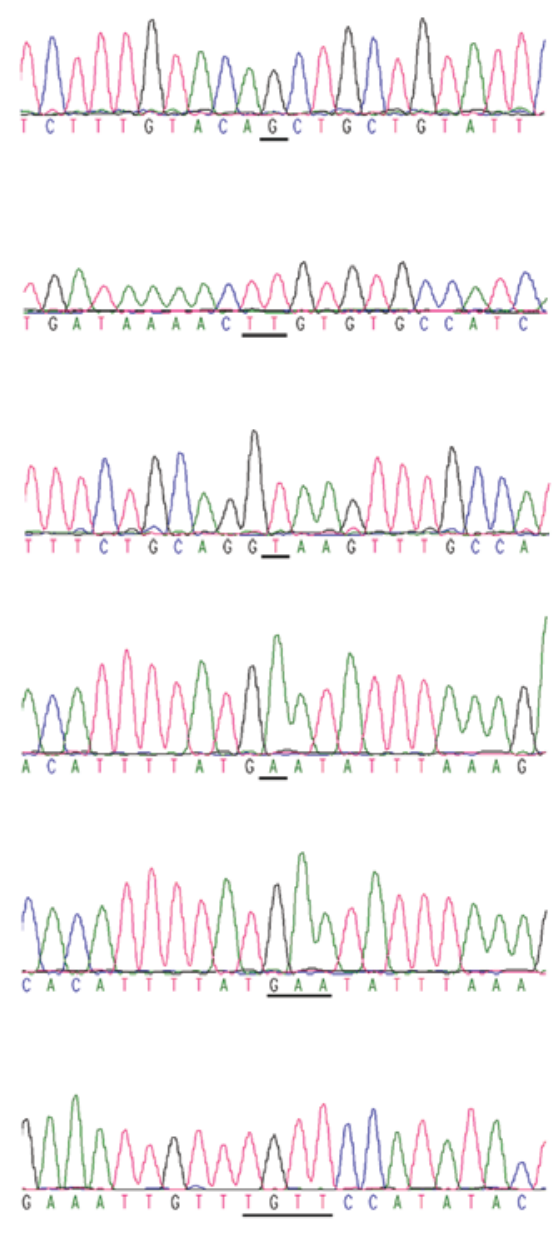

Figure 1. Sequence chromatography showed $C H M$ mutations. The proband numbers and associated pedigrees are listed on the left, followed by the hemizygous mutations in the six probands in the middle, and corresponding normal sequences on the right.

Board of the Zhongshan Ophthalmic. Genomic DNA was prepared from venous leukocytes.

Exome sequencing. Exome sequencing was performed by BGI Shenzhen, as previously described (14). The exome sequencing, genotype calling, and SNP calling were conducted as previously described (15). Exome capture was carried out using a NimbleGen SeqCap EZ Exome (44M) array (Roche, Basil, Switzerland). Exon-enriched DNA fragments were sequenced by the Illumina Genome Analyzer II (Illumina, Inc., San Diego, CA, USA). The average sequencing depth was set at 60 -fold. The SOAP aligner was used to align the sequencing reads to 
Table II. Hemizygous mutations in the $C H M$ gene and their associated clinical data.

\begin{tabular}{|c|c|c|c|c|c|c|c|c|c|c|c|}
\hline \multirow{2}{*}{$\begin{array}{l}\text { Proband } \\
\text { ID }\end{array}$} & \multicolumn{3}{|c|}{$\mathrm{CHM}$ variation } & \multirow[t]{2}{*}{ Gender } & \multicolumn{2}{|c|}{ Age (year) at } & \multirow{2}{*}{$\begin{array}{c}\text { First } \\
\text { symptom }\end{array}$} & \multirow{2}{*}{$\begin{array}{c}\text { Visual } \\
\text { acuity } \\
\text { (right; left) }\end{array}$} & \multirow{2}{*}{$\begin{array}{l}\text { Fundus } \\
\text { changes }\end{array}$} & \multicolumn{2}{|c|}{ ERG responses } \\
\hline & Nucleotide change & Effect & Database & & exam & onset & & & & rod & cone \\
\hline RP31 & c. $[703-1 \mathrm{G}>\mathrm{A}] ;[0]$ & SSA & Known [34] & M & 23 & 5 & NB & $0.3 ; 0.3$ & $\mathrm{AV}, \mathrm{CS}, \mathrm{BSP}$ & NA & NA \\
\hline RP229 & c.[558_559delTT];[0] & T186TfsX12 & Novel & M & 34 & 7 & NB & $0.1 ; 0.6$ & $\mathrm{AV}, \mathrm{CS}, \mathrm{BSP}$ & ND & ND \\
\hline RP263 & c. $[1166+2 \mathrm{~T}>\mathrm{G}] ;[0]$ & SSA & Novel & M & 22 & $\mathrm{EC}$ & NB & $0.7 ; 0.7$ & AV, WSP & ND & ND \\
\hline RP285 & c.[966delA];[0] & E322DfsX3 & Novel & M & 34 & $\mathrm{EC}$ & NB & $0.2 ; 0.3$ & SPP & NA & NA \\
\hline RP304 & c. $[964 \mathrm{G}>\mathrm{T}] ;[0]$ & E322* & Novel & M & 24 & $\mathrm{EC}$ & NB & $0.5 ; 0.5$ & BSP & NA & NA \\
\hline RP359 & c.[1584_1587delTGTT];[0] & F528FfsX8 & Known [34] & M & 21 & $\mathrm{EC}$ & NB & $0.4 ; 0.03$ & $\mathrm{AV}, \mathrm{CS}, \mathrm{BSP}$ & ND & ND \\
\hline
\end{tabular}

SSA, splicing site abolished; M, male; EC, early childhood; NB, night blindness; AV, attenuated vessels; CS, choroidal sclerosis; BSP, bone spicule-like pigmentation; WSP, wide-spread pigment deposit at retina; SPP, 'salt and pepper'-like pigment mottling; NA, not available; ND, not detectable.

UCSC hg19 (16,17). The likelihood of possible genotypes in target regions was calculated using SOAPsnp (18). Data were reviewed for all the genes known to be associated with hereditary retinal disease. Probands with a hemizygous mutation in the $C H M$ gene but without other causative mutations in the 61 of the 62 RP-associated genes were selected in this study.

Sanger sequencing. Sanger sequencing was used to verify the variants in $\mathrm{CHM}$ detected by exome sequencing. The fragments with variants found in patients were amplified by the polymerase chain reaction with the primers listed in Table I. The sequences of the amplicons were determined with an ABI Big Dye Terminator cycle sequencing kit v3.1 on an ABI3130 Genetic Analyzer (both from Applied Biosystems, Foster City, CA, USA). Sequencing results from patients and controls were aligned using the SeqManII program of the Lasergene package (DNAStar, Inc., Madison, WI, USA). Variants in available family members were also analyzed. Novel variants were then evaluated in 96 control individuals. The mutations were described in accordance with the nomenclature for the description of sequence variants (HGVS: http://www.hgvs. org/mutnomen/).

\section{Results}

Whole exome sequencing detected six hemizygous mutations in $C H M$ in six of the 157 families with RP. Sanger sequencing confirmed the six mutations in CHM (Fig. 1). Of the six, four were novel and two were known mutations (Table II). Four of the six resulted in truncation of the encoded proteins and the remaining two were predicted to eliminate the splicing sites (Table II). Potential pathogenic mutations in the 62 RP-associated genes were not detected in the six families.

For the patients with the CHM mutations, three were singleton cases without a family history of retinal degeneration while the remaining three had a family history consistent with an X-linked trait. All the probands had experienced night blindness since early childhood, with gradually reduced visual acuity later in life. Fundus observation revealed retinal degeneration with pigmentary disturbance. A review of the fundus images obtained showed fundus changes consistent with choroideremia (Fig. 2) although typical indications of choroideremia, such as chorioretinal scalloped atrophy in
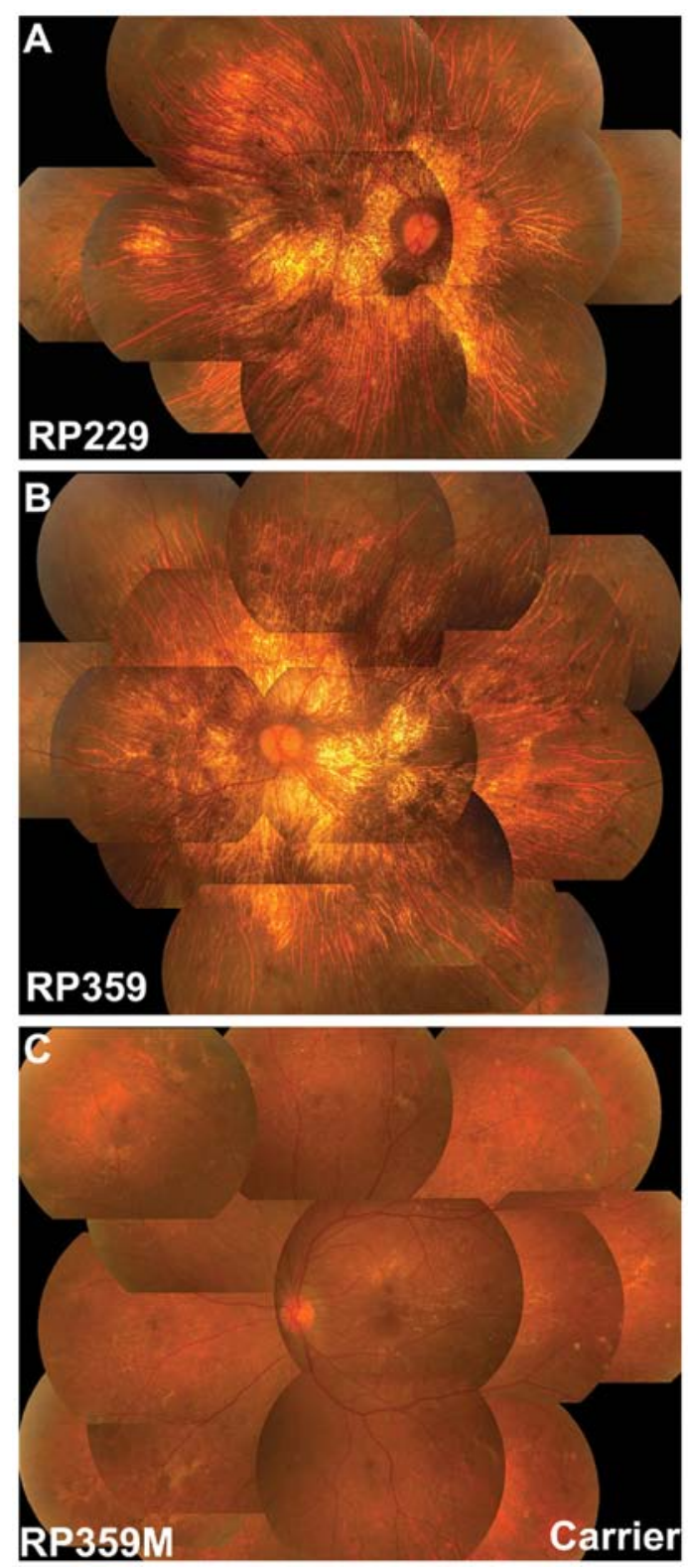

Figure 2. Images show fundus changes in two probands (RP229 and RP359) and the carrier mother (RP359M) of RP359. Attenuated retinal vessels, choroidal sclerosis, and pigment disturbance in the mid-peripheral retina are evident. Yellow crystalline-like spots are aggregated in the macular region and irregular mottled pigmentation occurred in the mid-periphery fundus in the carrier mother. 
the mid-peripheral fundus with preserved macula, was not recorded in these patients (Table II). Electroretinography recordings on three probands showed no appreciable responses of cones and rods in both eyes.

Available family members were analyzed in family RP359, where the mother (RP359M) of the proband had a heterozygous c.1584_1587delTGTT mutation. The mother had normal visual acuity without night blindness, but had a number of crystalline-like spots in the macular area and irregular retinal dystrophy in the mid-peripheral retina (Fig. 2). Electroretinography showed normal rod responses and mildly reduced cone responses.

\section{Discussion}

The present study identified six hemizygous $C H M$ mutations in six of the 15 unrelated families with initial diagnosis of $\mathrm{RP}$, initially analyzed by exome sequencing and confirmed by Sanger sequencing. All six mutations resulted in truncation (or loss of function) that is generally observed in $C H M$ mutations. Initial clinical diagnosis of the six probands was RP while subsequent re-evaluation of the clinical data suggested atypical form of choroideremia.

Mutations in CHM are known to cause choroideremia alone (9,19-37). At least 147 mutations in CHM have been previously reported in patients with choroideremia, including 2 missense mutations, 39 nonsense mutations, 25 splicing mutations, 32 small deletions, 9 small insertions, 5 small indels, 31 gross deletions, 1 gross insertions/duplications, and 3 complex rearrangements, based on the HGMD ${ }^{\circledR}$ Professional 2012.4 (https://portal.biobase-international. $\mathrm{com} / \mathrm{hgmd} / \mathrm{pro} / \mathrm{gene}$.php?gene=CHM). All but two of these $(21,38)$ are loss of function mutations. The six mutations identified in this study include 1 nonsense, 3 small deletion, and 2 splicing mutations, all of which are loss of function mutations.

Choroideremia and RP share several common features, such as night blindness, constriction of the visual field, gradually reduced visual acuity, and retinal degeneration, and may be confused with each other (39). Previous studies have suggested that 'about $6 \%$ of individuals diagnosed with RP-related disorders actually have choroideremia' (39), while about one quarter of clinically diagnosed choroideremia may actually be other diseases, including RP (40). Choroideremia, referring to the absence (-eremia) of choroid, is an X-linked disease characterized by chorioretinal scalloped atrophy initiated from the mid-peripheral fundus, with preservation of the macula (20,40-42). However, these types of typical fundus changes for choroideremia may not yet have developed at the first visit to the ophthalmologists. Considering the great variability in the appearance of the fundus in RP, choroideremia without a typical fundus appearance may easily be diagnosed as RP.

The typical manifestation for choroideremia [i.e., chorioretinal scalloped atrophy with preservation of the macula $(42,43)]$, was not found in the six probands with $C H M$ mutations in the present study. However, the fundus changes of the six probands with $C H M$ mutations were also atypical compared to those seen in classic RP. Retinal pigmentary degeneration with choroidal sclerosis has been visualized, not only in choroideremia, but also in severe retinitis pigmentosa with the PROMI mutation (44) or in Bietti crystalline corneoretinal dystrophy with the CYP4V2 mutation (45). The six patients in the present study with atypical fundus changes may be misdiagnosed as $\mathrm{RP}$ if phenotypic variation of choroideremia is not obvious and systemic fundus examination is not performed.

In summary, six mutations leading to the truncation of CHM were identified in six of $157(4 \%)$ unrelated patients with initial diagnosis of RP. The results of this study suggest that CHM should be included as a candidate gene for atypical RP. Additionally, choroideremia may be misdiagnosed as RP. These findings emphasize that genes known to cause one form of retinal degeneration may also be ideal candidates for other forms of retinal degeneration, particularly for those with overlapping phenotypes.

\section{Acknowledgements}

The authors would like to thank the patients for their participation. This study was supported by the National Natural Science Foundation of China (U1201221 to Q.Z.), '985 project' of Sun Yat-sen University, and the Fundamental Research Funds of State Key Laboratory of Ophthalmology.

\section{References}

1. Hartong DT, Berson EL and Dryja TP: Retinitis pigmentosa. Lancet 368: 1795-1809, 2006.

2. den Hollander AI, Black A, Bennett J and Cremers FP: Lighting a candle in the dark: advances in genetics and gene therapy of recessive retinal dystrophies. J Clin Invest 120: 3042-3053, 2010.

3. Xu Y, Guan L, Shen T, Zhang J, Xiao X, Jiang H, Li S, Yang J, Jia X, Yin Y, Guo X, Wang J and Zhang Q: Mutations of 60 known causative genes in 157 families with retinitis pigmentosa based on exome sequencing. Hum Genet (In press).

4. Züchner S, Dallman J, Wen R, et al: Whole-exome sequencing links a variant in DHDDS to retinitis pigmentosa. Am J Hum Genet 88: 201-206, 2011.

5. Tucker BA, Scheetz TE, Mullins RF, et al: Exome sequencing and analysis of induced pluripotent stem cells identify the cilia-related gene male germ cell-associated kinase (MAK) as a cause of retinitis pigmentosa. Proc Natl Acad Sci USA 108: E569-E576, 2011.

6. Peluso I, Conte I, Testa F, et al: The ADAMTS18 gene is responsible for autosomal recessive early onset severe retinal dystrophy. Orphanet J Rare Dis 8: 16, 2013.

7. Webb TR, Parfitt DA, Gardner JC, et al: Deep intronic mutation in OFD1, identified by targeted genomic next-generation sequencing, causes a severe form of X-linked retinitis pigmentosa (RP23). Hum Mol Genet 21: 3647-3654, 2012.

8. Wang Y, Guo L, Cai SP, et al: Exome sequencing identifies compound heterozygous mutations in CYP4V2 in a pedigree with retinitis pigmentosa. PLoS One 7: e33673, 2012.

9. Cremers FP, van de Pol DJ, van Kerkhoff LP, Wieringa B and Ropers HH: Cloning of a gene that is rearranged in patients with choroideraemia. Nature 347: 674-677, 1990.

10. Rak A, Pylypenko O, Niculae A, Pyatkov K, Goody RS and Alexandrov K: Structure of the Rab7:REP-1 complex: insights into the mechanism of Rab prenylation and choroideremia disease. Cell 117: 749-760, 2004.

11. Seabra MC, Brown MS and Goldstein JL: Retinal degeneration in choroideremia: deficiency of rab geranylgeranyl transferase. Science 259: 377-381, 1993.

12. Berger W, Kloeckener-Gruissem B and Neidhardt J: The molecular basis of human retinal and vitreoretinal diseases. Prog Retin Eye Res 29: 335-375, 2010.

13. Zhang Q, Zulfiqar F, Xiao X, et al: Severe autosomal recessive retinitis pigmentosa maps to chromosome 1p13.3-p21.2 between D1S2896 and D1S457 but outside ABCA4. Hum Genet 118: 356-365, 2005. 
14. Xiao X, Li S, Guo X and Zhang Q: A novel locus for autosomal dominant congenital motor nystagmus mapped to 1q31-q32.2 between D1S2816 and D1S2692. Hum Genet 131: 697-702, 2012.

15. Li Y, Vinckenbosch N, Tian G, et al: Resequencing of 200 human exomes identifies an excess of low-frequency non-synonymous coding variants. Nat Genet 42: 969-972, 2010.

16. Li R, Li Y, Kristiansen K and Wang J: SOAP: short oligonucleotide alignment program. Bioinformatics 24: 713-714, 2008

17. Li R, Yu C, Li Y, et al: SOAP2: an improved ultrafast tool for short read alignment. Bioinformatics 25: 1966-1967, 2009.

18. Li R, Li Y, Fang X, et al: SNP detection for massively parallel whole-genome resequencing. Genome Res 19: 1124-1132, 2009.

19. Chi JY, MacDonald IM and Hume S: Copy number variant analysis in CHM to detect duplications underlying choroideremia. Ophthalmic Genet 34: 229-233, 2013.

20. Coussa RG and Traboulsi EI: Choroideremia: a review of general findings and pathogenesis. Ophthalmic Genet 33: 57-65, 2012.

21. Esposito G, De Falco F, Tinto N, et al: Comprehensive mutation analysis (20 families) of the choroideremia gene reveals a missense variant that prevents the binding of REP1 with Rab geranylgeranyl transferase. Hum Mutat 32: 1460-1469, 2011.

22. Forsythe P, Maguire A, Fujita R, Moen C, Swaroop A and Bennett J: A carboxy-terminal truncation of 99 amino acids resulting from a novel mutation (Arg555 $\rightarrow$ stop) in the CHM gene leads to choroideremia. Exp Eye Res 64: 487-490, 1997.

23. Fujiki K, Hotta Y, Hayakawa M, et al: REP-1 gene mutations in Japanese patients with choroideremia. Graefes Arch Clin Exp Ophthalmol 237: 735-740, 1999.

24. Garcia-Hoyos M, Lorda-Sanchez I, Gómez-Garre P, et al: New type of mutations in three Spanish families with choroideremia. Invest Ophthalmol Vis Sci 49: 1315-1321, 2008.

25. Hotta Y, Fujiki K, Hayakawa M, et al: A hemizygous A to CC base change of the CHM gene causing choroideremia associated with pinealoma. Graefes Arch Clin Exp Ophthalmol 235: 653-655, 1997

26. Iino Y, Fujimaki T, Fujiki K and Murakami A: A novel mutation $(967-970+2)$ delAAAGGT in the choroideremia gene found in a Japanese family and related clinical findings. Jpn J Ophthalmol 52: 289-297, 2008.

27. Itabashi T, Wada Y, Kawamura M, Sato $\mathrm{H}$ and Tamai M: Clinical features of Japanese families with a 402delT or a 555-556delAG mutation in choroideremia gene. Retina 24: 940-945, 2004.

28. McTaggart KE, Tran M, Mah DY, Lai SW, Nesslinger NJ and MacDonald IM: Mutational analysis of patients with the diagnosis of choroideremia. Hum Mutat 20: 189-196, 2002

29. Nesslinger N, Mitchell G, Strasberg P and MacDonald IM: Mutation analysis in Canadian families with choroideremia. Ophthalmic Genet 17: 47-52, 1996.

30. Perez-Cano HJ, Garnica-Hayashi RE and Zenteno JC: CHM gene molecular analysis and X-chromosome inactivation pattern determination in two families with choroideremia. Am J Med Genet A 149A: 2134-2140, 2009.
31. Ponjavic V, Abrahamson M, Andreasson S, et al: Phenotype variations within a choroideremia family lacking the entire CHM gene. Ophthalmic Genet 16: 143-150, 1995.

32. Sankila EM, Tolvanen R, van den Hurk JA, Cremers FP and de la Chapelle A: Aberrant splicing of the CHM gene is a significant cause of choroideremia. Nat Genet 1: 109-113, 1992.

33. Schwartz M, Rosenberg T, van den Hurk JA, van de Pol DJ and Cremers FP: Identification of mutations in Danish choroideremia families. Hum Mutat 2: 43-47, 1993.

34. van Bokhoven H, Schwartz M, Andréasson S, et al: Mutation spectrum in the CHM gene of Danish and Swedish choroideremia patients. Hum Mol Genet 3: 1047-1051, 1994.

35. Yip SP, Cheung TS, Chu MY, et al: Novel truncating mutations of the CHM gene in Chinese patients with choroideremia. Mol Vis 13: 2183-2193, 2007

36. Zhou Q, Liu L, Xu F, et al: Genetic and phenotypic characteristics of three Mainland Chinese families with choroideremia. Mol Vis 18: 309-316, 2012.

37. Lin Y, Liu X, Luo L, et al: Molecular analysis of the choroideremia gene related clinical findings in two families with choroideremia. Mol Vis 17: 2564-2569, 2011.

38. Sergeev YV, Smaoui N, Sui R, et al: The functional effect of pathogenic mutations in Rab escort protein 1. Mutat Res 665: 44-50, 2009.

39. MacDonald IM, Smaoui N and Seabra MC: Choroideremia. In: GeneReviews ${ }^{\circledR}$ [Internet]. Pagon RA, Adam MP, Bird TD, Dolan CR, Fong CT, Smith RJH and Stephens K (ed). University of Washington, Seattle, pp1993-2014, 2003.

40. Lee TK, McTaggart KE, Sieving PA, et al: Clinical diagnoses that overlap with choroideremia. Can J Ophthalmol 38: 364-372, 2003.

41. Roberts MF, Fishman GA, Roberts DK, et al: Retrospective, longitudinal, and cross sectional study of visual acuity impairment in choroideraemia. Br J Ophthalmol 86: 658-662, 2002.

42. Mura M, Sereda C, Jablonski MM, MacDonald IM and Iannaccone A: Clinical and functional findings in choroideremia due to complete deletion of the CHM gene. Arch Ophthalmol 125: 1107-1113, 2007.

43. MacDonald IM, Russell L and Chan CC: Choroideremia: new findings from ocular pathology and review of recent literature. Surv Ophthalmol 54: 401-407, 2009.

44. Zhang Q, Zulfiqar F, Xiao X, et al: Severe retinitis pigmentosa mapped to $4 \mathrm{p} 15$ and associated with a novel mutation in the PROM1 gene. Hum Genet 122: 293-299, 2007.

45. Xiao X, Mai G, Li S, Guo X and Zhang Q: Identification of CYP4V2 mutation in 21 families and overview of mutation spectrum in Bietti crystalline corneoretinal dystrophy. Biochem Biophys Res Commun 409: 181-186, 2011. 